\title{
QUALITY OF SERVICE ASSESSMENT IN LTE NETWORKS WITH A LIMITED NUMBER OF USERS
}

\author{
Ndayikunda Juven, \\ University of Bujumbura, Bujumbura, Burundi, \\ juvndayi@mail.ru
}

KEYWORDS: Internet of Things (IoT), LTE, eMTM, dynamic allocation, rate of lost requests, resource blocks, NB-IoT, bandwidth

\begin{abstract}
Narrow Band Internet of Things (NB-IoT) is the latest cellular radio access technology that was based on LTE technology and implemented as part of the 3GPP 3GPP 3GPP for Low-power Wide-area Network (LPWAN). The paper considers the features of NB-IoT standards based on the characteristics of the physical and channel layers of NBIoT technology based on release 14-15 of the 3GPP group. The aim of this work is also to build and study a model for serving heterogeneous traffic in an isolated cell of the LTE standard, which supports the functionality of the Internet of Things IoT (Internet of Things), in particular eMTC (enhanced Machine Type Communication). The constructed mathematical model takes into account the heterogeneity of the arrival of requests and their dependence on the number of users of cellular services. Depending on the number of sources, incoming requests are described by Poisson or Engset models. The studied model makes it possible to dynamically allocate resources that have LTE.
\end{abstract}

DOI: $10.36724 / 2664-066 X-2020-6-2-\mid 4-19$

\section{INTRODUCTION}

The current stage of development of infocommunication networks requires solving urgent problems of analyzing the characteristics of the quality of service of incoming requests. Over the past few years, there has been a rapid growth in the number of telecommunication devices. According to Cisco forecasts, the number of devices connected to the Internet is growing rapidly and already exceeds the world's population [1,2]. These include eMTC devices and various IoT sensors [6]. The increase in the number of devices connected to the Internet has made it necessary to develop new methods for assessing the quality of communication. The existing communication infrastructure is used to transfer the data traffic. Such networks are often deployed in locations where wired communication is limited. Wired Internet connection of telecommunication devices is not always possible in some areas. For the transmission of video information, voice messages, etc. in such places where there is no possibility of using wired communication at all, a wireless communication network is used. The LTE standard in the last 10 years has been a leader in the field of mobile communications.

In 2014, the 3rd Generation Partnership Project (3GPP) began development on the Internet of Things standard i.e. provision of services to users using the technology "energy efficient long-range network LPWAN (Low-power Wide-area Network)". In the LPWAN category, there are licensed technologies such as M1 (LTE-M) and unlicensed technologies, for example. Long Range (LoRa), SigFox, Ingenu, etc. In this paper, we consider servicing requests from a finite number of users, which are surveillance cameras and requests from traditional LTE devices.

This paper examines the features of the NB-IoT standard in comparison with the characteristics of LTE technology at the physical layer. When writing this article, the materials of the LTE Rel.14, 15 specifications were used, published in December 2016 - November 2017. 


\section{Formulation of the problem}

Let's consider a cellular communication of the LTE standard providing information transfer to the analytical center. The available radio resources of an LTE cell in the uplink direction are measured in terms of their smallest granularity, called a channel or unit resource. The total number of unit resources is a linear function of the number of RB (Resource Blocks).

In the investigated cell of the LTE standard, there are a number of LTE devices and IoT devices connected to the base station. LTE devices and IoT devices are sources of requests [7]. For simplicity of calculations, it is assumed that its base station is located in the center of the cell, all claims equally use the base station resource allocated to one claim, regardless of the distance between the base station and the terminal. The selection of active devices is performed in a circular cyclic scheme (Round Robin) as shown in [3].

Let $\mathrm{s}$ be the number of video surveillance devices with high quality of service. To transmit video information of such devices, it is necessary to use a large number of channel units.

Table 1

\section{Number of resource blocks depending} on the channel width

\begin{tabular}{|l|l|l|l|l|l|l|}
\hline Channel width, $\mathrm{MHz}$ & 1,4 & 3 & 5 & 10 & 1 & \\
\hline $\begin{array}{l}\text { Number of resource } \\
\text { blocks }\end{array}$ & 6 & 15 & 25 & 50 & & \\
\hline
\end{tabular}

Let me denote by $v$ - the total number of units of the required channel resources (the total number of channel units). Let us recall that in the fourth generation mobile communication network built according to the LTE standard, the resource is resource blocks that depend on the frequency band (Table 1).

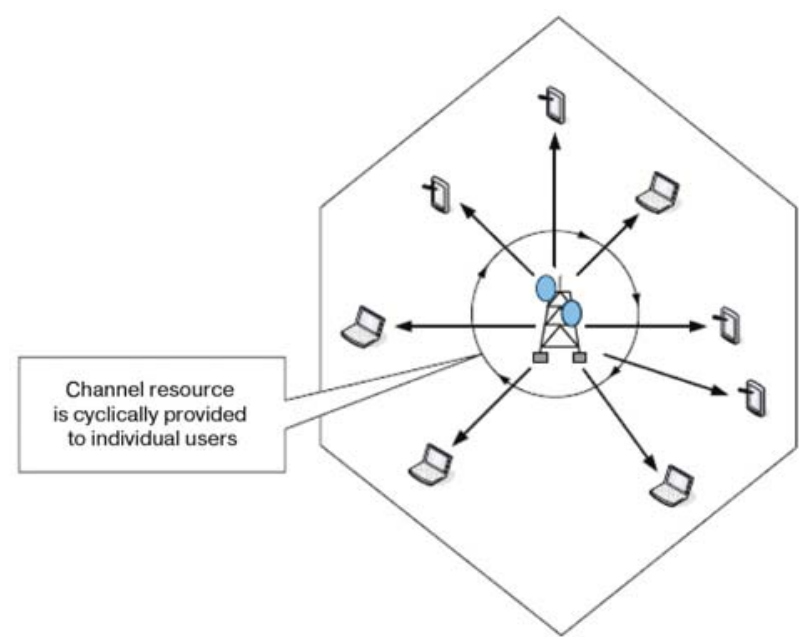

Figure 1. Scheme of active subscribers cyclic service in a cell

Denote by $\beta$ exponential distribution parameter of time interval before sending the corresponding request from one source from final group of users.
Intensity of IoT requests receipt depends on the number of active video surveillance devices and is equal to $\left(s-i_{1}\right) \beta$, where $i_{1}$ is the number of requests from origin in target group of users in service.

Denote by $b_{1}$ - the number of channel units needed to service one request created by a limited number of video surveillance devices. The intensity of the proposed traffic is determined by the following formula [4]:

$$
a_{1}=\frac{s \beta}{\mu_{1}+\beta}
$$

where $\mu_{1}$ is a parameter of exponential distribution of service time interval for requests from a finite user group source.

The constructed model takes into account the heterogeneity of incoming traffic flows, i.e. dependence of receipt intensity and requests processing on the traffic type. Model receives the second type of requests originating from a large number of LTE devices. Based on this assumption, a change in their numbers does not greatly affect intensity of incoming requests. In this case, we can use the Poisson model to describe the process of incoming requests.

The arrival of requests from traditional LTE devices obeys Poisson's law with an intensity $\lambda$. To service the incoming request, $b_{2}$ channel units are used. The suggested traffic intensity is determined using the following expression:

$$
a_{2}=\frac{\lambda}{\mu_{2}}
$$

where $\mu_{2}-$ parameter of request servicing time intervalexponential distribution.

\section{Markov process and quality of service characteristics}

Let $i_{1}(t)$ be the number of requests for the transmission of video information in service at a point in time $t \geq 0$ and $t \geq 0$ - the number of requests from traditional LTE devices in service at a point in time $t \geq 0$. The change in the state of the model is described by a random Markov process $r(t)=\left(i_{1}(t), i_{2}(t)\right)$ defined on the state space $S$, consisting of vectors $\left(i_{1}, i_{2}\right)$ with components:

$$
\begin{aligned}
& i_{1}=0,1, \ldots, \min \left(s_{1},\left\lfloor\frac{v}{b_{1}}\right\rfloor\right) \\
& i_{2}=0,1, \ldots,\left\lfloor\frac{v-i_{1} b_{1}}{b_{2}}\right\rfloor .
\end{aligned}
$$


Let us denote $i$ by the number of cell resource units occupied in the state of servicing video traffic $\left(i_{1}, i_{2}\right)$ and traffic from traditional LTE devices and is defined as the total product of the number of requests being serviced at a point in time $t \geq 0$ and the number of channel units required to transmit the corresponding requests $\left(p\left(i_{1}, i_{2}\right)\right.$ - stationary probability of the model $\left(i_{1}, i_{2}\right)$ state).

It is convenient to compose the equations of statistical equilibrium of the system following the following rule: the total incoming flow of probabilities into the state $\left(i_{1}, i_{2}\right)$ must be equal to the total outgoing flow of probabilities from the state of the carrot process $\left(i_{1}, i_{2}\right)$ model $r(t)$ in its stationary mode. Using the indicator of the function, we represent all the equations of the system of equations of equilibrium of states in one relation, which makes it convenient for the implementation of iterative algorithms for solving the corresponding system.

The system of equilibrium equations connecting the unnormalized probabilities has the form:

$$
\begin{aligned}
& P\left(i_{1}, i_{2}\right)\left\{\left(s-i_{1}\right) \beta I\left(i+b_{1} \leq v\right)+i_{1} \mu_{1} I\left(i_{1}>0\right)+\right. \\
& \left.+\lambda I\left(i+b_{2} \leq v\right)+i_{2} \mu_{2} I\left(i_{2}>0\right)\right\}= \\
& =P\left(i_{1}-1, i_{2}\right)\left(s-i_{1}+1\right) \beta I\left(i_{1}>0\right)+ \\
& +P\left(i_{1}, i_{2}-1\right) \lambda I\left(i_{2}>0\right)+ \\
& +P\left(i_{1}+1, i_{2}\right)\left(i_{1}+1\right) \mu_{1} I\left(i+b_{1} \leq v, i_{1}+1 \leq s\right)+ \\
& +P\left(i_{1}, i_{2}+1\right)\left(i_{2}+1\right) \mu_{2} I\left(i+b_{2} \leq v\right) \text {. }
\end{aligned}
$$

Let us denote by $\pi_{1}$ and $\pi_{2}$ the shares of the time of resource occupancy at the moment of receipt of an application for servicing video traffic and traffic from traditional LTE devices, respectively. Let us denote by

$\pi_{1, c}$ and $\pi_{2, c}$ the shares of lost requests for the transmission of video traffic and traffic from endless traditional LTE devices, respectively.

Let us denote by $m_{1}$ and $m_{2}$ - the average number of resource units occupied in the state of $\left(i_{1}, i_{2}\right)$ servicing video traffic and traffic from traditional LTE devices, respectively.

The average number of requests for video traffic and traffic transmission from traditional LTE devices will be denoted by $y_{1}$ and $y_{2}$ respectively.

$$
\begin{aligned}
& \pi_{k}=\sum_{\left(i_{1}, i_{2}\right) \in S \mid i+b_{k}>v} p\left(i_{1}, i_{2}\right) ; \quad k=1,2 ; \\
& \pi_{1, c}=\frac{\sum_{\left(i_{1}, i_{2}\right) \in S \mid i+b_{1}>v} p\left(i_{1}, i_{2}\right)\left(s-i_{2}\right) \beta}{\sum_{\left\{\left(i_{1}, i_{2}\right) \in S\right\}} p\left(i_{1}, i_{2}\right)\left(s-i_{2}\right) \beta} ;
\end{aligned}
$$

$$
\begin{aligned}
& \pi_{2, c}=\pi_{2} ; \\
& m_{k}=\sum_{\left(i_{1}, i_{2}\right) \in S} p\left(i_{1}, i_{2}\right) i_{k} b_{k} ; \quad k=1,2 ; \\
& y_{k}=\sum_{\left(i_{1}, i_{2}\right) \in S} p\left(i_{1}, i_{2}\right) i_{k} ; \quad k=1,2 .
\end{aligned}
$$

To assess the listed service quality indicators, it is necessary to find the value $p\left(i_{1}, i_{2}\right)$ by solving the equilibrium equation system (1) by the iterative GaussSeidel method [5].

\section{LTE base station resource with NB-IoT functionality. LTE frame structure}

In the past 10 years, there has been a rapid growth in the number of wireless communications. According to Cisco's forecast, the number of devices connected to the Internet is growing rapidly $(10 \%$ CAGR (Compound annual growth rate)) and already exceeds the world's population [2-3]. A significant proportion of such devices are focused on transmitting a small amount of information (NB-IoT files) at a rate of $\sim 250 \mathrm{kbit} / \mathrm{s}$ and $\sim 226.7 \mathrm{kbit} / \mathrm{s}$ via the downlink and uplink, respectively.

There is a need to connect a huge number of devices to the Internet. Therefore, the 3GPP consortium has implemented the NB-IoT narrowband Internet of Things standard as a solution to this problem. NB-IoT is classified as one of the licensed low power, long range, and low cost cellular technologies for wide area LPWANs based on LTE technology. There are other licensed technologies in the LPWAN category, such as M1 (LTE-M), and unlicensed technologies, for example. Long Range (LoRa), SigFox, Ingenu, etc.

The radio interface of the LTE technology is based on the Orthogonal Frequency-Division Multiplexing OFDM technology. In order to increase the data transfer rate, LTE networks use the technology of multi-antenna systems MIMO (Multiple Input Multiple Output).

OFDM technology assumes the transmission of a wideband signal through the independent modulation of narrowband subcarriers of the form $s_{k}=a_{k} \sin \left[2 \pi\left(f_{0}+k \Delta f\right)\right]$ that are located with a certain frequency step $\Delta f$. The OFDM symbol contains a set of modulated subcarriers, a payload field and a so-called Cyclic Prefix CP. In LTE standard, the step between subcarriers $\Delta \mathrm{f}$ is $15 \mathrm{kHz}$, which corresponds to an OFDM symbol duration of $66.7 \mu \mathrm{s}$.

Each subscriber unit in a slot is assigned a specific range of channel resources in the frequency time domain (Figure 2). The length of an LTE frame is $10 \mathrm{~ms}$ and each frame is divided into 10 subframes of $1 \mathrm{~ms}$ each. One subframe contains two slots. The duration of each slot is $0.5 \mathrm{~ms}$. Depending on the cyclic prefix configuration, each slot has 7 and 6 OFDM symbols. 


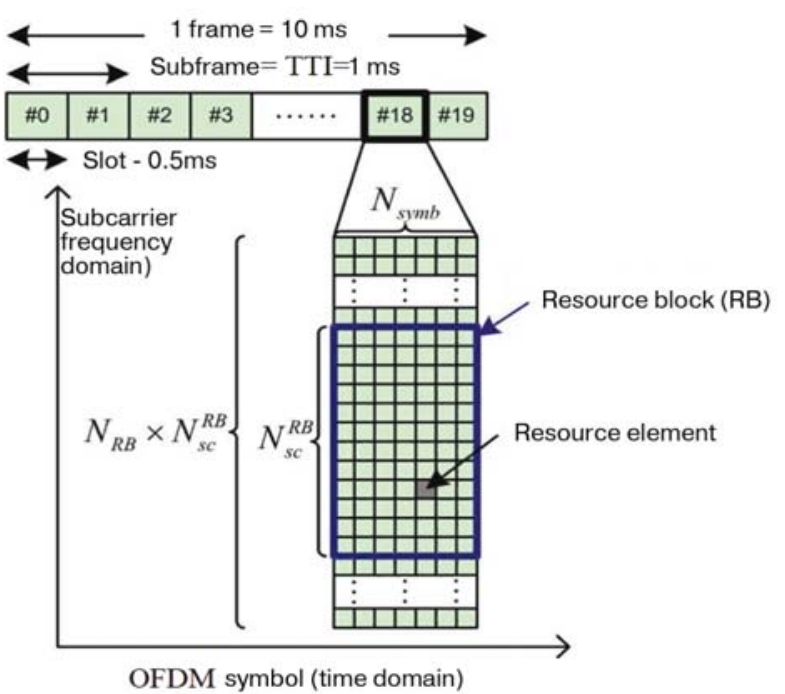

Figure 2. LTE frame structure

In the frequency domain, each channel resource consists of Resource Blocks. In turn, each resource block consists of 12 adjacent subcarriers, occupying a bandwidth of $180 \mathrm{kHz}$ (Fig. 1). The number of resource blocks depends on the channel bandwidth and varies from 6 to 110 resource blocks [2]
The resource block is the main resource unit for scheduling data transmission in the radio interface and is the minimum resource element allocated to the subscriber unit by the base station scheduler.

\section{Features of the technology of narrowband Internet of things NB-IoT}

NB-IoT devices can operate at lower signal levels, hence higher noise levels. Another advantage is the battery saving [5]. NB-IoT technology is designed for short message transmission only, not for transmission of audio / video content or large files.

This section discusses the features of the physical layer of NB-IoT technology. Also presented is a stack of NB-IoT protocols, based on modern PHY and MAC levels, in order to identify the knowledge gap and determine the direction of development of narrowband Internet of things technology.

Infrastructure of NB-IoT standard network is deployed on top of the existing LTE infrastructure. The 3GPP consortium offers three options for deploying NB-IoT networks: narrow-band Internet of things on the guard band (NB-IoT Guard Band), in-band (In Band), and stand-alone (Stand Alone) (Fig. 3).

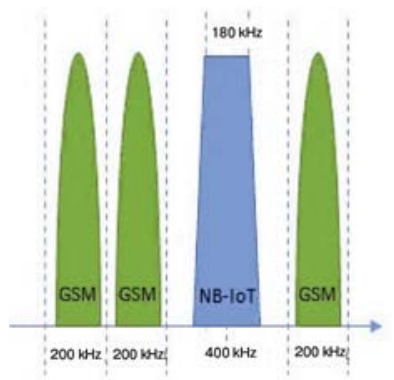

a) Autonomous

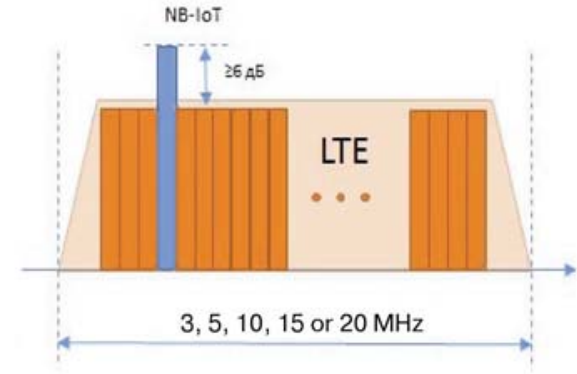

b) In-band

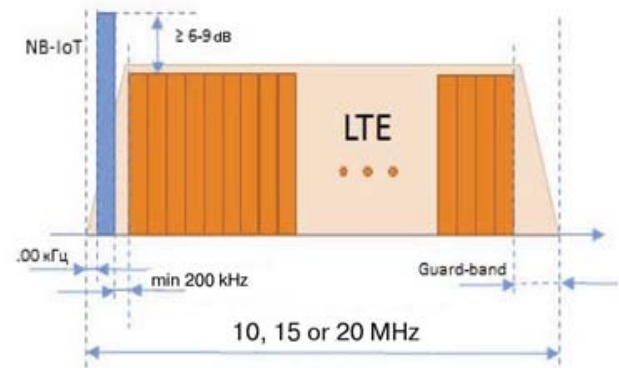

c) On the protective strip

Figure 3. Deployment options for narrowband Internet of things

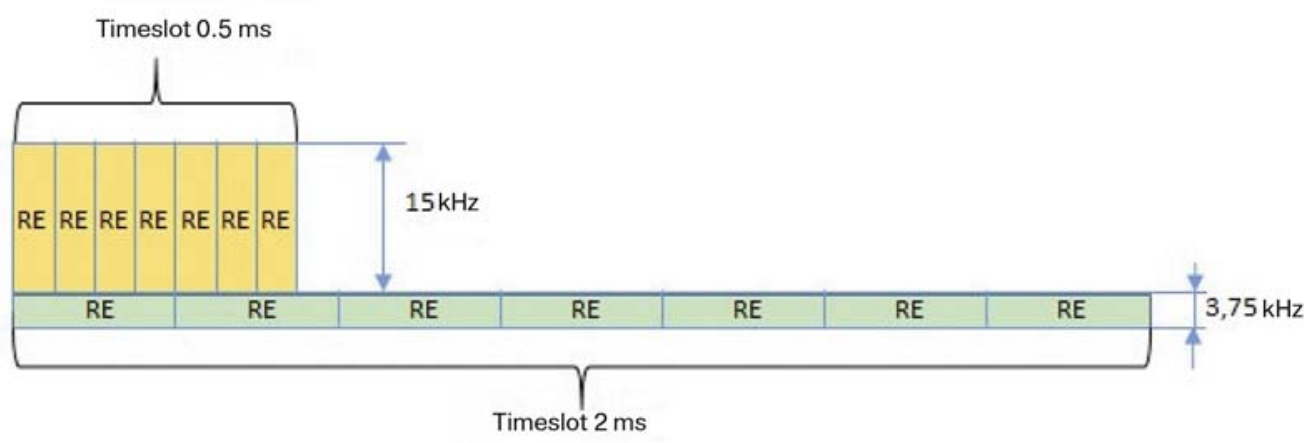

Figure 4. Resource element 


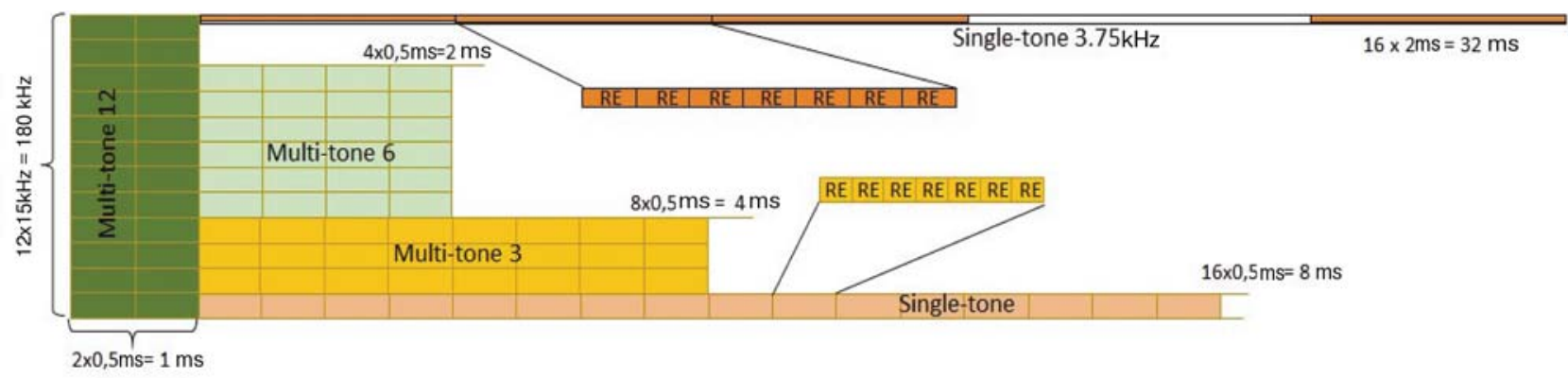

Figure 5. Single resource RU [9]

\section{Physical channels and signals}

NB-IoT standard adopts the same frame structure as LTE [6-8]. One superframe consists of 1024 frames, which in turn contain 10 subframes each. One subframe contains two time slots of $0.5 \mathrm{~ms}$ each in the time domain. In the frequency domain, either 12 subcarriers are used in each $0.5 \mathrm{~ms}$ slot, or 48 subcarriers are used with a $2 \mathrm{~ms}$ slot at a spacing frequency of $3.75 \mathrm{kHz}$ in the uplink.

NB-IoT uses the same protocol stack as LTE. However, some design changes at both the PHY and MAC levels have been introduced to support massive long distance connections with MCL (Maximun Coupling Loss) up to $20 \mathrm{~dB}$ compared to traditional technologies such as LTE, GSM and GPRS [1].

At the physical layer of NB-IoT, the same technologies are used as in LTE. OFDM and SC-FDMA technologies are used in the NB-IoT standard to generate signals on the transmission of information in the downlink and uplink, respectively. However, the resource scheduling block in NB-IoT is a resource element RE (Resource Element) (or tone) instead of PRB (Physical Resource Block), allowing NB-IoT devices to transmit a signal on a single subcarrier at $15 \mathrm{kHz}$, which makes it possible to serve multiple users in a frequency band $180 \mathrm{kHz}$.

Signal transmission in a narrow band on a single subcarrier of $15 \mathrm{kHz}$, and the same at a lower frequency of $3.75 \mathrm{kHz}$, can significantly increase the spectral density of the signal and, therefore, the signal-to-noise ratio increases, which is very important for subscriber devices that have much less powerful transmitters than the base station.

\section{Support for multi-tone transmission - Multi-Tone Transmission}

To connect mass devices to one base station, NB-IoT distributes resource units (RU - Resource Units) among several user devices, in contrast to LTE, where the entire resource unit is allocated to one user in the uplink [1].

The uplink communication uses the SC-FDMA multiplexing scheme. The frequency spacing between subcarriers is $3.75 \mathrm{kHz}$ or $15 \mathrm{kHz}$. On the downlink, NBIoT uses $15 \mathrm{kHz}$ with OFDM, just like LTE. With $15 \mathrm{kHz}$ spacing, NB-IoT can allocate both single-tone $(8 \mathrm{~ms})$ and multi-tone (3 tones, 6 tones, and 12 tones) to different users in $4 \mathrm{~ms}, 2 \mathrm{~ms}$ and $1 \mathrm{~ms}$ durations, respectively. With $3.75 \mathrm{kHz}$, only single-tone is supported with 48 subframes of $32 \mathrm{~ms}$ duration. RU is another larger brick from which transport blocks (Transport blocks, TB), assigned to the user, are formed.

\section{Conclusion}

This article discusses dynamic resource allocation for sharing real-time IoT traffic streams, packet data, and traffic from traditional LTE devices over radio resources of an LTE cell. In the constructed model, two types of flows were considered. The first type of traffic represents streams coming from sources from the end user group and the second from traditional LTE devices. Depending on the number of sources, incoming requests are described by Poisson or Engset models.

All the random parameters used in the model have an exponential distribution with corresponding mean values. And also in the work, a model for assessing the quality of service requests in a mobile communication network of the LTE standard is built, taking into account the heterogeneity of incoming traffic flows.

The analysis process of applications joint service in isolated cell of the LTE standard, which supports the IoT functionality, in particular, eMTM, is carried out. Using the constructed model, the main indicators of the quality of service are determined on the basis of obtained values of the limiting probabilities of model stationary states.

Investigated model allows to dynamically distribute the available resource between incoming requests. The constructed model provides operators with additional opportunities to improve quality of LTE cellular network.

The LTE base station with NB-IoT functionality uses DCI (Downlink Control Information) to transmit downlink / uplink scheduling overhead to NB-IoT. Then, based on the scheduling information received, the NB-IoT user equipment (UE) determines the deployment mode of the NB-IoT network (autonomous mode, in-band mode or in the guard interval), as well as cell identification and 
finds out which resource elements are already in use in LTE.

Thus, the UE can map the PDCCH and PDSCH symbols into available resource elements. For example, in the downlink, NPDCCH is transmitted by aggregating narrowband control elements (entry 0 and entry 1 ), where entry 0 is transmitted by subcarriers 0 through 5 and entry 1 using subcarriers 6 through 11 in a subframe.

In addition, DCI can be multiplexed in one subframe, or one DCI can be mapped in one subframe, according to the aggregation level used. NB-IoT uses limited BPSK and QPSK modulation schemes with support for only one antenna, both in uplink and downlink.

\section{References}

1. Collins Burton Mwakwata, Hassan Malik, Muhammad Mahtab Alam, Yannick Le Moullec, Sven Parand, Shahid Mumtaz. Narrowband Internet of Things (NB-IoT): From Physical (PHY) and Media Access Control (MAC) Layers Perspectives. Sensors (Basel). 2019 Jun 8.

2. 3GPP ETSI TS 136300 V14.3.0. LTE; Evolved Universal Terrestrial Radio Access (E-UTRA) and Evolved Universal Terrestrial Radio Access Network (E-UTRAN); Overall description. 2017. 347 p.

3. 3GPP TS 36.101 V15.0. Evolved Universal Terrestrial Radio Access (E-UTRA). User Equipment (UE) radio transmission and reception. 2017. 1547 p.

4. Ryzhkov A.E. Development of NB-IoT technology. Proceedings of educational institutions of communication. 2017. Vol. 3. No. 4. P. 94-101.

5. Salman, L., Salman, S., Jahangirian, S., Abraham, M.; German, F., Blair C., Krenz P. Energy efficient IoT-based smart home. IEEE 3rd World Forum on Internet of Things (WF-IoT), Reston, VA, USA, 12-14 December 2016.
6. Beyene Y.D., Jantti R., Tirkkonen O., Ruttik K., Iraji S., Larmo A.; Tirronen T.; Torsner J. NB-IoT Technology Overview and Experience from Cloud-RAN Implementation. IEEE Wirel. Commun. 2017, 24, pp. 26-32.

7. $\mathrm{Xu}$ T.; Darwazeh I. Non-Orthogonal Narrowband Internet of Things: A Design for Saving Bandwidth and Doubling the Number of Connected Devices. IEEE Internet Things J. 2018, 5, 2120-2129.

8. Adhikary A., Lin X., Wang Y.E. Performance Evaluation of NB-IoT Coverage. IEEE 84th Vehicular Technology Conference (VTC-Fall), Montreal, QC, Canada, 18 21 September 2016.

9. Ilnur Fauziev. NB-IoT: how does it work? Part 1. URL: https://habr.com/ru/company/ru_mts/blog/430496/ (date of access 10.12.2019).

10. Cisco Visual Networking Index: Forecast and Methodology, 2017-2022, February, 2019.

11. Stepanov S.N. Teletraffic theory: concepts, models, applications. Series "Theory and Practice of Infocommunications". Moscow: Hotline - Telecom, 2015. 868 p.

12. Huawei Global Industry Vision 2025, 2018.

13. Stepanov S.N., Romanov A.M., Osiya D.L. Construction and analysis of a data transmission model on an access line from a finite group of subscribers. T-Comm. 2015. Vol. 9. No. 9. P. 29-34.

14. Stepanov S.N., Stepanov M.S., Malikova E.E.,

15. Tsogbadrakh A., Ndayikunda J. Construction and analysis of a generalized resource sharing model for LTE technologies with NB-IoT functionality. T-Comm. 2018. Vol. 12. No. 12. P. 71-77.

16. Victor Alekseev. Quectel modules with NB-IoT and eMTC support. Wireless technology. No.2. 2018. P. 9-16.

17. Begishev V., Petrov V., Samuylov A., Moltchanov D., Andreev S., Koucheryavy Y., Samouylov K. Resource Allocation and Sharing for Heterogeneous Data Collection over Conventional 3GPP LTE and Emerging NB-IoT Technologies. Computer Communications. 2018. Vol. 120. No. 2. P. 93-101. 